\title{
The obstacle problem for non-coercive equations with lower order term and $L^{1}$-data
}

Jun Zheng ${ }^{1 *}$ (D)

\section{"Correspondence:}

zhengjun2014@aliyun.com

'School of Mathematics, Southwest

Jiaotong University, Chengdu, China

\section{Abstract}

The aim of this paper is to study the obstacle problem associated with an elliptic operator having degenerate coercivity, a low order term, and $L^{1}$-data. We prove the existence of an entropy solution to the obstacle problem and show its continuous dependence on the $L^{1}$-data in $W^{1, q}(\Omega)$ with some $q>1$.

MSC: Primary 35J87; 35J70; secondary 35B45

Keywords: Obstacle problem; Non-coercive equation; Entropy solution; L $L^{1}$-data; Lower order term

\section{Introduction}

\subsection{Problem setting and main result}

Let $\Omega$ be a bounded domain in $\mathbb{R}^{N}(N \geq 2), 1<p<+\infty$, and $\theta \geq 0$. Given functions $g, \psi \in W^{1, p}(\Omega) \cap L^{\infty}(\Omega)$ and data $f \in L^{1}(\Omega)$, the aim of this paper is to study the obstacle problem for nonlinear non-coercive elliptic equations with lower order term, governed by the operator

$$
A u=-\operatorname{div} \frac{a(x, \nabla u)}{(1+|u|)^{\theta(p-1)}}+b|u|^{r-2} u
$$

where $b>0$ is a constant, and $a: \Omega \times \mathbb{R}^{N} \rightarrow \mathbb{R}^{N}$ is a Carathéodory function, satisfying the following conditions:

$$
\begin{aligned}
& a(x, \xi) \cdot \xi \geq \alpha|\xi|^{p}, \\
& |a(x, \xi)| \leq \beta\left(j(x)+|\xi|^{p-1}\right), \\
& (a(x, \xi)-a(x, \eta))(\xi-\eta)>0, \\
& |a(x, \xi)-a(x, \zeta)| \leq \gamma \begin{cases}|\xi-\zeta|^{p-1}, & \text { if } 1<p<2, \\
(1+|\xi|+|\zeta|)^{p-2}|\xi-\zeta|, & \text { if } p \geq 2\end{cases}
\end{aligned}
$$

for almost every $x$ in $\Omega$ and for every $\xi, \eta, \zeta$ in $\mathbb{R}^{N}$ with $\xi \neq \eta$, where $\alpha, \beta, \gamma>0$ are constants, and $j$ is a nonnegative function in $L^{p^{\prime}}(\Omega)$.

(c) The Author(s) 2019. This article is distributed under the terms of the Creative Commons Attribution 4.0 International License (http://creativecommons.org/licenses/by/4.0/), which permits unrestricted use, distribution, and reproduction in any medium, provided you give appropriate credit to the original author(s) and the source, provide a link to the Creative Commons license, and indicate if changes were made. 
If $f$ has a fine regularity, e.g., $f \in W^{-1, p^{\prime}}(\Omega)$, the obstacle problem corresponding to $(f, \psi, g)$ can be formulated in terms of the inequality

$$
\begin{aligned}
& \int_{\Omega} \frac{a(x, \nabla u)}{(1+|u|)^{\theta(p-1)}} \cdot \nabla(u-v) \mathrm{d} x+\int_{\Omega} b|u|^{r-2} u(u-v) \mathrm{d} x \\
& \quad \leq \int_{\Omega} f(u-v) \mathrm{d} x, \quad \forall v \in K_{g, \psi} \cap L^{\infty}(\Omega),
\end{aligned}
$$

whenever $1 \leq r<p$ and the convex subset

$$
K_{g, \psi}=\left\{v \in W^{1, p}(\Omega) ; v-g \in W_{0}^{1, p}(\Omega), v \geq \psi \text {, a.e. in } \Omega\right\}
$$

is nonempty. However, if $f \in L^{1}(\Omega)$, (6) is not well-defined. Following [1, 3, 5, 19] etc., we are led to the more general definition of a solution to the obstacle problem, using the truncation function

$$
T_{s}(t)=\max \{-s, \min \{s, t\}\}, \quad s, t \in \mathbb{R} .
$$

Definition 1 An entropy solution of the obstacle problem associated with operator $A$ and functions $(f, \psi, g)$ with $f \in L^{1}(\Omega)$ is a measurable function $u$ such that $u \geq \psi$ a.e. in $\Omega, \frac{a(x, \nabla u)}{(1+|u|)^{\theta(p-1)}} \in\left(L^{1}(\Omega)\right)^{N},|u|^{r-1} \in L^{1}(\Omega)$, and, for every $s>0, T_{s}(u)-T_{s}(g) \in W_{0}^{1, p}(\Omega)$ and

$$
\begin{aligned}
& \int_{\Omega} \frac{a(x, \nabla u)}{(1+|u|)^{\theta(p-1)}} \cdot \nabla\left(T_{s}(u-v)\right) \mathrm{d} x+\int_{\Omega} b|u|^{r-2} u T_{s}(u-v) \mathrm{d} x \\
& \quad \leq \int_{\Omega} f T_{s}(u-v) \mathrm{d} x, \quad \forall v \in K_{g, \psi} \cap L^{\infty}(\Omega) .
\end{aligned}
$$

Observe that no global integrability condition is required on $u$ nor on its gradient in the definition. As pointed out in [3, 8], if $T_{s}(u) \in W^{1, p}(\Omega)$ for all $s>0$, then there exists a unique measurable vector field $U: \Omega \rightarrow \mathbb{R}^{N}$ such that $\nabla\left(T_{s}(u)\right)=\chi_{\{|| u \mid<s\}} U$ a.e. in $\Omega$, $s>0$, which, in fact, coincides with the standard distributional gradient of $\nabla u$ whenever $u \in W^{1,1}(\Omega)$.

Before stating the main result, we make some basic assumptions throughout this paper, i.e., without special statements, we always assume that

$$
2-\frac{1}{N}<p<N, \quad 1 \leq r<p, \quad 0 \leq \theta<\min \left\{\frac{N}{N-1}-\frac{1}{p-1}, \frac{p-r}{p-1}\right\}, \quad b>0,
$$

and $\psi, g \in W^{1, p}(\Omega) \cap L^{\infty}(\Omega)$ with $(\psi-g)^{+} \in W_{0}^{1, p}(\Omega)$ such that $K_{g, \psi} \neq \emptyset$. The following theorem is the main result obtained in this paper.

Theorem 1 Let $f \in L^{1}(\Omega)$. Then there exists at least one entropy solution $u$ of the obstacle problem associated with $(f, \psi, g)$. In addition, $u$ depends continuously on $f$, i.e., if $f_{n} \rightarrow f$ in $L^{1}(\Omega)$ and $u_{n}$ is a solution to the obstacle problem associated with $\left(f_{n}, \psi, g\right)$, then

$$
u_{n} \rightarrow u \quad \text { in } W^{1, q}(\Omega), \forall q \in \begin{cases}\left(\frac{N(r-1)}{N+r-1}, \frac{N(p-1)(1-\theta)}{N-1-\theta(p-1)}\right), & \text { if } \frac{2 N-1}{N-1} \leq r<p, \\ \left(1, \frac{N(p-1)(1-\theta)}{N-1-\theta(p-1)}\right), & \text { if } 1 \leq r<\min \left\{\frac{2 N-1}{N-1}, p\right\} .\end{cases}
$$




\subsection{Some comments and remarks}

Consider the Dirichlet boundary value problem having a form

$$
\begin{cases}-\operatorname{div} \frac{\left.|\nabla u|\right|^{p-2} \nabla u}{\left(1+\left.|u|\right|^{\theta(p-1)}\right.}+b u=f, & \text { in } \Omega, \\ u=0, & \text { on } \partial \Omega,\end{cases}
$$

with $p>1, \theta \in(0,1], b \geq 0, f \in L^{1}(\Omega)$. The item $-\operatorname{div} \frac{|\nabla u|^{\mid-2} \nabla u}{\left(1+|u|^{\theta(p-1)}\right.}$ may not be coercive when $u$ tends to infinity. Due to this fact, the classical methods used to prove the existence of a solution for elliptic equations, e.g., [14], cannot be applied even if $b=0$ and the data $f$ is regular. Moreover, $\frac{|\nabla u|^{p-2} \nabla u}{\left(1+|u|^{\theta(p-1)}\right.}, u$ and $f$ are only in $L^{1}(\Omega)$, not in $W^{-1, p^{\prime}}(\Omega)$. All these characteristics prevent us from employing the duality argument [17] or nonlinear monotone operator theory [18] directly.

To overcome these difficulties, "cutting" the non-coercivity term and using the technique of approximation, a pseudomonotone and coercive differential operator on $W_{0}^{1, p}(\Omega)$ can be applied to establish a priori estimates on approximating solutions. As a result, existence of solutions, or entropy solutions, can be obtained by taking limitation for $f \in L^{m}(\Omega), m \geq 1$, and $b>0$ due to the almost everywhere convergence of gradients of the approximating solutions, see, e.g., $[4,6,9-11,15]$ (see also $[1,2,7,12,13,16]$ for $b=0)$. However, there is little literature that considers regularities for entropy solutions of obstacle problems governed by (1) and functions $(f, \psi, g)$ with $f \in L^{1}(\Omega)$, except [19], in which the authors considered the obstacle problem (7) with $b=0$ and $L^{1}$-data.

Motivated by the study on the non-coercive elliptic equations (9) and the problem considered in [19], in this paper, we consider the obstacle problem governed by (1) and functions $(f, \psi, g)$ with $f \in L^{1}(\Omega)$. By the truncation method used in [8] and [19], we prove the existence of an entropy solution and show its continuous dependence on the $L^{1}$-data in $W^{1, q}(\Omega)$ with some $q \in(1, p)$.

In the following, we give some remarks on our main result and inequalities that will be needed in the proofs. Some notations are provided at the end of this subsection.

\section{Remark 1}

(i) $0 \leq \theta<\min \left\{\frac{N}{N-1}-\frac{1}{p-1}, \frac{p-r}{p-1}\right\} \Rightarrow r-1<(1-\theta)(p-1)<\frac{N(p-1)(1-\theta)}{N-1-\theta(p-1)}$. Therefore Theorem 1 guarantees $|u|^{r-1} \in L^{1}(\Omega)$, and the second integration in (7) makes sense.

(ii) We will show that $\frac{a(x, \nabla u)}{(1+|u|)^{\theta(p-1)}} \in\left(L^{1}(\Omega)\right)^{N}$ in Proposition 4. Therefore, the first integration in (7) makes sense.

(iii) $\left(\frac{N(r-1)}{N+r-1}, \frac{N(p-1)(1-\theta)}{N-1-\theta(p-1)}\right) \subset\left(1, \frac{N(p-1)(1-\theta)}{N-1-\theta(p-1)}\right)$ if $\frac{2 N-1}{N-1} \leq r<p$. Indeed, $\theta<\frac{p-r}{p-1}+\frac{p(r-1)}{N(p-1)} \Leftrightarrow \frac{N(p-1)(1-\theta)}{N-1-\theta(p-1)}>\frac{N(r-1)}{N+r-1}$, while $\frac{2 N-1}{N-1} \leq r$ gives $\frac{N(r-1)}{N+r-1} \geq 1$. Thus $u_{n} \rightarrow u$ in $W^{1, q}(\Omega)$ for all $q \in\left(1, \frac{N(p-1)(1-\theta)}{N-1-\theta(p-1)}\right)$.

(iv) $r-1<\frac{N q}{N-q}$. Indeed, by $1 \leq r<\frac{2 N-1}{N-1}$, there holds $r-1<\frac{N}{N-1}<\frac{N q}{N-q}$ for any $q>1$, particularly, for $q \in\left(1, \frac{N(p-1)(1-\theta)}{N-1-\theta(p-1)}\right)$. For $r \geq \frac{2 N-1}{N-1}$, it suffices to note that $q>\frac{N(r-1)}{N+r-1} \Leftrightarrow r-1<\frac{N q}{N-q}$.

(v) $q<p$. Indeed, $0 \leq \theta<\frac{N}{N-1}-\frac{1}{p-1}<\frac{N-1}{p-1} \Rightarrow \frac{N(p-1)(1-\theta)}{N-1-\theta(p-1)}<p$. 
Remark 2 Checking proofs in this paper (e.g., setting $r=1$ ), one may find that, for $b=0$,

(8) holds with

$$
u_{n} \rightarrow u \quad \text { in } W^{1, q}(\Omega), \forall q \in\left(1, \frac{N(p-1)(1-\theta)}{N-1-\theta(p-1)}\right),
$$

which is the same as [19, Theorem 1]. Thus, Theorem 1 can be seen as an extension of [19, Theorem 1].

Notations $\|u\|_{p}:=\|u\|_{L^{p}(\Omega)}$ is the norm of $u$ in $L^{p}(\Omega)$, where $1 \leq p \leq \infty$. $\|u\|_{1, p}:=$ $\|u\|_{W^{1, p}(\Omega)}$ is the norm of $u$ in $W^{1, p}(\Omega)$, where $1<p<\infty \cdot p^{\prime}:=\frac{p}{p-1}$ with $1<p<\infty$. $\{u>s\}:=$ $\{x \in \Omega ; u(x)>s\} .\{u \leq s\}:=\Omega \backslash\{u>s\} .\{u<s\}:=\{x \in \Omega ; u(x)<s\} .\{u \geq s\}:=\Omega \backslash\{u<s\}$. $\{u=s\}:=\{x \in \Omega ; u(x)=s\} .\{t \leq u<s\}:=\{u \geq t\} \cap\{u<s\}$. For a measurable set $E$ in $\mathbb{R}^{N}$, $|E|:=\mathcal{L}^{N}(E)$, where $\mathcal{L}^{N}$ is the Lebesgue measure of $\mathbb{R}^{N}$. For a real-valued function $u$, $u^{+}=\max \{u, 0\}, u^{-}=(-u)^{+}$. Without special statements, positive integers are denoted by $n, h, k, k_{0}, K . C$ is a positive constant, which may be different from each other.

\section{Lemmas on entropy solutions}

It is worthy to note that, for any smooth function $f_{n}$, there exists at least one solution to the obstacle problem (6). Indeed, one can proceed exactly as in $[1,11]$ to obtain $W^{1, p}$-solutions due to assumptions (2)-(4) on $a$ and $r-1<p$. These solutions, in particular, are also entropy solutions. In this section, using the method of [8] and [19], we establish several auxiliary results on convergence of sequences of entropy solutions when $f_{n} \rightarrow f$ in $L^{1}(\Omega)$.

Lemma 2 Let $v_{0} \in K_{g, \psi} \cap L^{\infty}(\Omega)$, and let $u$ be an entropy solution of the obstacle problem associated with $(f, \psi, g)$. Then we have

$$
\int_{\{|u|<t\}} \frac{|\nabla u|^{p}}{(1+|u|)^{\theta(p-1)}} \mathrm{d} x \leq C\left(1+t^{r}\right), \quad \forall t>0,
$$

where $C$ is a positive constant depending only on $\alpha, \beta, p, r, b,\|j\|_{p^{\prime}},\left\|\nabla v_{0}\right\|_{p},\left\|v_{0}\right\|_{\infty}$, and $\|f\|_{1}$.

Proof Take $v_{0}$ as a test function in (7). For $t$ large enough such that $t-\left\|v_{0}\right\|_{\infty}>0$, we get

$$
\begin{aligned}
\int_{\left\{\left|v_{0}-u\right|<t\right\}} \frac{a(x, \nabla u) \cdot \nabla u}{(1+|u|)^{\theta(p-1)}} \mathrm{d} x \leq & \int_{\left\{\left|\nu_{0}-u\right|<t\right\}} \frac{a(x, \nabla u) \cdot \nabla v_{0}}{(1+|u|)^{\theta(p-1)}} \mathrm{d} x \\
& +\int_{\Omega}\left(f-b|u|^{r-2} u\right) T_{t}\left(u-v_{0}\right) \mathrm{d} x .
\end{aligned}
$$

We estimate each integration in the right-hand side of (11). It follows from (3) and Young's inequality with $\varepsilon>0$ that

$$
\begin{aligned}
\int_{\left\{\left|\nu_{0}-u\right|<t\right\}} \frac{a(x, \nabla u) \cdot \nabla v_{0}}{(1+|u|)^{\theta(p-1)}} \mathrm{d} x \leq & \int_{\left\{\left|v_{0}-u\right|<t\right\}} \frac{\beta\left(|j|+|\nabla u|^{p-1}\right) \cdot\left|\nabla v_{0}\right|}{(1+|u|)^{\theta(p-1)}} \mathrm{d} x \\
\leq & \int_{\left\{\left|\nu_{0}-u\right|<t\right\}} \frac{\beta \varepsilon\left(|j|^{p^{\prime}}+|\nabla u|^{p}\right)}{(1+|u|)^{\theta(p-1)}} \mathrm{d} x \\
& +\int_{\left\{\left|\nu_{0}-u\right|<t\right\}} \frac{\beta C(\varepsilon)\left|\nabla v_{0}\right|^{p}}{(1+|u|)^{\theta(p-1)}} \mathrm{d} x
\end{aligned}
$$




$$
\begin{aligned}
\leq & \varepsilon \int_{\left\{\left|\nu_{0}-u\right|<t\right\}} \frac{|\nabla u|^{p}}{(1+|u|)^{\theta(p-1)}} \mathrm{d} x \\
& +C\left(\|j\|_{p^{\prime}}^{p^{\prime}}+\left\|\nabla v_{0}\right\|_{p}^{p}\right), \\
-\int_{\Omega} b|u|^{r-2} u T_{t}\left(u-v_{0}\right) \mathrm{d} x= & -\int_{\left\{\left|u-v_{0}\right| \leq t\right\}} b|u|^{r-2} u T_{t}\left(u-v_{0}\right) \mathrm{d} x \\
& -\int_{\left\{\left|u-v_{0}\right|>t\right\}} b|u|^{r-2} u T_{t}\left(u-v_{0}\right) \mathrm{d} x .
\end{aligned}
$$

Note that on the set $\left\{\left|u-v_{0}\right| \leq t\right\}$,

$$
\left.|| u\right|^{r-2} u T_{t}\left(u-v_{0}\right)|\leq t| t+\left.\left\|v_{0}\right\|_{\infty}\right|^{r-1} \leq C\left(1+t^{r}\right),
$$

where $C$ is a constant depending only on $r,\left\|v_{0}\right\|_{\infty}$.

On the set $\left\{\left|u-v_{0}\right|>t\right\}$, we have $|u| \geq t-\left\|v_{0}\right\|_{\infty}>0$, thus $u$ and $T_{t}\left(u-v_{0}\right)$ have the same sign. It follows

$$
-\int_{\left\{\left|u-v_{0}\right|>t\right\}} b|u|^{r-2} u T_{t}\left(u-v_{0}\right) \mathrm{d} x \leq 0
$$

Combining (13)-(15), we get

$$
\begin{aligned}
-\int_{\Omega} b|u|^{r-2} u T_{t}\left(u-v_{0}\right) \mathrm{d} x & \leq C\left(1+t^{r}\right) \\
\int_{\left\{\left|v_{0}-u\right|<t\right\}} \frac{|\nabla u|^{p}}{(1+|u|)^{\theta(p-1)}} \mathrm{d} x & \leq C\left(\|j\|_{p^{\prime}}^{p^{\prime}}+\left\|\nabla v_{0}\right\|_{p}^{p}+t\|f\|_{1}+1+t^{r}\right) \\
& \leq C\left(1+t^{r}\right) .
\end{aligned}
$$

Replacing $t$ with $t+\left\|v_{0}\right\|_{\infty}$ in (17) and noting that $\{|u|<t\} \subset\left\{\left|v_{0}-u\right|<t+\left\|v_{0}\right\|_{\infty}\right\}$, one may obtain the desired result.

In the rest of this section, let $\left\{u_{n}\right\}$ be a sequence of entropy solutions of the obstacle problem associated with $\left(f_{n}, \psi, g\right)$ and assume that

$$
f_{n} \rightarrow f \quad \text { in } L^{1}(\Omega) \quad \text { and } \quad\left\|f_{n}\right\|_{1} \leq\|f\|_{1}+1 .
$$

Lemma 3 There exists a measurable function $u$ such that $u_{n} \rightarrow u$ in measure, and $T_{k}\left(u_{n}\right) \rightarrow T_{k}(u)$ weakly in $W^{1, p}(\Omega)$ for any $k>0$. Thus $T_{k}\left(u_{n}\right) \rightarrow T_{k}(u)$ strongly in $L^{p}(\Omega)$ and a.e. in $\Omega$.

Proof Let $s, t$, and $\varepsilon$ be positive numbers. One may verify that, for every $m, n \geq 1$,

$$
\begin{aligned}
\mathcal{L}^{N}\left(\left\{\left|u_{n}-u_{m}\right|>s\right\}\right) \leq & \mathcal{L}^{N}\left(\left\{\left|u_{n}\right|>t\right\}\right)+\mathcal{L}^{N}\left(\left\{\left|u_{m}\right|>t\right\}\right) \\
& +\mathcal{L}^{N}\left(\left\{\left|T_{k}\left(u_{n}\right)-T_{k}\left(u_{m}\right)\right|>s\right\}\right),
\end{aligned}
$$

and

$$
\mathcal{L}^{N}\left(\left\{\left|u_{n}\right|>t\right\}\right)=\frac{1}{t^{p}} \int_{\left\{\left|u_{n}\right|>t\right\}} t^{p} \mathrm{~d} x \leq \frac{1}{t^{p}} \int_{\Omega}\left|T_{t}\left(u_{n}\right)\right|^{p} \mathrm{~d} x
$$


Due to $v_{0}=g+(\psi-g)^{+} \in K_{g, \psi} \cap L^{\infty}(\Omega)$, by Lemma 2, one has

$$
\int_{\Omega}\left|\nabla T_{t}\left(u_{n}\right)\right|^{p} \mathrm{~d} x=\int_{\left\{\left|u_{n}\right|<t\right\}}\left|\nabla u_{n}\right|^{p} \mathrm{~d} x \leq C(1+t)^{\theta(p-1)}\left(1+t^{r}\right) .
$$

Note that $T_{t}\left(u_{n}\right)-T_{t}(g) \in W_{0}^{1, p}(\Omega)$. By (19), (20), and Poincaré's inequality, for every $t>$ $\|g\|_{\infty}$ and for some positive constant $C$ independent of $n$ and $t$, there holds

$$
\begin{aligned}
\mathcal{L}^{N}\left(\left\{\left|u_{n}\right|>t\right\}\right) & \leq \frac{1}{t^{p}} \int_{\Omega}\left|T_{t}\left(u_{n}\right)\right|^{p} \mathrm{~d} x \\
& \leq \frac{2^{p-1}}{t^{p}} \int_{\Omega}\left|T_{t}\left(u_{n}\right)-T_{t}(g)\right|^{p} \mathrm{~d} x+\frac{2^{p-1}}{t^{p}}\|g\|_{p}^{p} \\
& \leq \frac{C}{t^{p}} \int_{\Omega}\left|\nabla T_{t}\left(u_{n}\right)-\nabla T_{t}(g)\right|^{p} \mathrm{~d} x+\frac{2^{p-1}}{t^{p}}\|g\|_{p}^{p} \\
& \leq \frac{C}{t^{p}} \int_{\Omega}\left|\nabla T_{t}\left(u_{n}\right)\right|^{p} \mathrm{~d} x+\frac{C}{t^{p}}\|g\|_{1, p}^{p} \\
& \leq \frac{C\left(1+t^{r+\theta(p-1)}\right)}{t^{p}} .
\end{aligned}
$$

Since $0 \leq \theta<\frac{p-r}{p-1}$, there exists $t_{\varepsilon}>0$ such that

$$
\mathcal{L}^{N}\left(\left\{\left|u_{n}\right|>t\right\}\right)<\frac{\varepsilon}{3}, \quad \forall t \geq t_{\varepsilon}, \forall n \geq 1
$$

Now we have as in (19)

$$
\begin{aligned}
\mathcal{L}^{N}\left(\left\{\left|T_{t_{\varepsilon}}\left(u_{n}\right)-T_{t_{\varepsilon}}\left(u_{m}\right)\right|>s\right\}\right) & =\frac{1}{s^{p}} \int_{\left\{\left|T_{t_{\varepsilon}}\left(u_{n}\right)-T_{t_{\varepsilon}}\left(u_{m}\right)\right|>s\right\}} s^{p} \mathrm{~d} x \\
& \leq \frac{1}{s^{p}} \int_{\Omega}\left|T_{t_{\varepsilon}}\left(u_{n}\right)-T_{t_{\varepsilon}}\left(u_{m}\right)\right|^{p} \mathrm{~d} x .
\end{aligned}
$$

Using (20) and the fact that $T_{t}\left(u_{n}\right)-T_{t}(g) \in W_{0}^{1, p}(\Omega)$ again, we see that $\left\{T_{t_{\varepsilon}}\left(u_{n}\right)\right\}$ is a bounded sequence in $W^{1, p}(\Omega)$. Thus, up to a subsequence, $\left\{T_{t_{\varepsilon}}\left(u_{n}\right)\right\}$ converges strongly in $L^{p}(\Omega)$. Taking into account (22), there exists $n_{0}=n_{0}\left(t_{\varepsilon}, s\right) \geq 1$ such that

$$
\mathcal{L}^{N}\left(\left\{\left|T_{t_{\varepsilon}}\left(u_{n}\right)-T_{t_{\varepsilon}}\left(u_{m}\right)\right|>s\right\}\right)<\frac{\varepsilon}{3}, \quad \forall n, m \geq n_{0} .
$$

Combining (18), (21), and (23), we obtain

$$
\mathcal{L}^{N}\left(\left\{\left|u_{n}-u_{m}\right|>s\right\}\right)<\varepsilon, \quad \forall n, m \geq n_{0}
$$

Hence $\left\{u_{n}\right\}$ is a Cauchy sequence in measure, and therefore there exists a measurable function $u$ such that $u_{n} \rightarrow u$ in measure. The remainder of the lemma is a consequence of the fact that $\left\{T_{k}\left(u_{n}\right)\right\}$ is a bounded sequence in $W^{1, p}(\Omega)$.

Proposition 4 There exist a subsequence of $\left\{u_{n}\right\}$ and a measurable function $u$ such that, for each q given in (8), we have

$$
u_{n} \rightarrow u \text { strongly in } W^{1, q}(\Omega)
$$


Furthermore, if $0 \leq \theta<\min \left\{\frac{1}{N-p+1}, \frac{N}{N-1}-\frac{1}{p-1}, \frac{p-r}{p-1}\right\}$, then

$$
\frac{a\left(x, \nabla u_{n}\right)}{\left(1+\left|u_{n}\right|\right)^{\theta(p-1)}} \rightarrow \frac{a(x, \nabla u)}{(1+|u|)^{\theta(p-1)}} \quad \text { strongly in }\left(L^{1}(\Omega)\right)^{N}
$$

To prove Proposition 4, we need two preliminary lemmas.

Lemma 5 There exist a subsequence of $\left\{u_{n}\right\}$ and a measurable function $u$ such that, for each q given in (8), we have $u_{n} \rightarrow u$ weakly in $W^{1, q}(\Omega)$, and $u_{n} \rightarrow u$ strongly in $L^{q}(\Omega)$.

Proof Let $k>0$ and $n \geq 1$. Define $D_{k}=\left\{\left|u_{n}\right| \leq k\right\}$ and $B_{k}=\left\{k \leq\left|u_{n}\right|<k+1\right\}$. Using Lemma 2 with $v_{0}=g+(\psi-g)^{+}$, we get

$$
\int_{D_{k}} \frac{\left|\nabla u_{n}\right|^{p}}{\left(1+\left|u_{n}\right|\right)^{\theta(p-1)}} \mathrm{d} x \leq C\left(1+k^{r}\right)
$$

where $C$ is a positive constant depending only on $\alpha, \beta, b, p, r,\|j\|_{p^{\prime}},\|f\|_{1},\left\|\nabla v_{0}\right\|_{p}$, and $\left\|v_{0}\right\|_{\infty}$.

Using the function $T_{k}\left(u_{n}\right)$ for $k>\left\{\|g\|_{\infty},\|\psi\|_{\infty}\right\}$, as a test function for the problem associated with $\left(f_{n}, \psi, g\right)$, we obtain

$$
\begin{aligned}
& \int_{\Omega} \frac{a\left(x, \nabla u_{n}\right) \cdot \nabla\left(T_{1}\left(u_{n}-T_{k}\left(u_{n}\right)\right)\right)}{\left(1+\left|u_{n}\right|\right)^{\theta(p-1)}} \mathrm{d} x+\int_{\Omega} b\left|u_{n}\right|^{r-2} u_{n} T_{1}\left(u_{n}-T_{k}\left(u_{n}\right)\right) \mathrm{d} x \\
& \quad \leq \int_{\Omega} f_{n} T_{1}\left(u_{n}-T_{k}\left(u_{n}\right)\right) \mathrm{d} x
\end{aligned}
$$

which and (2) give

$$
\int_{B_{k}} \frac{\alpha\left|\nabla u_{n}\right|^{p}}{\left(1+\left|u_{n}\right|\right)^{\theta(p-1)}} \mathrm{d} x+\int_{\Omega} b\left|u_{n}\right|^{r-2} u_{n} T_{1}\left(u_{n}-T_{k}\left(u_{n}\right)\right) \mathrm{d} x \leq\left\|f_{n}\right\|_{1} \leq\|f\|_{1}+1 .
$$

Note that on the set $\left\{\left|u_{n}\right| \geq k+1\right\}, u_{n}$ and $T_{1}\left(u_{n}-T_{k}\left(u_{n}\right)\right)$ have the same sign. Then

$$
\begin{aligned}
\int_{\Omega}\left|u_{n}\right|^{r-2} u_{n} T_{1}\left(u_{n}-T_{k}\left(u_{n}\right)\right) \mathrm{d} x= & \int_{D_{k}}\left|u_{n}\right|^{r-2} u_{n} T_{1}\left(u_{n}-T_{k}\left(u_{n}\right)\right) \mathrm{d} x \\
& +\int_{B_{k}}\left|u_{n}\right|^{r-2} u_{n} T_{1}\left(u_{n}-T_{k}\left(u_{n}\right)\right) \mathrm{d} x \\
& +\int_{\left\{\left|u_{n}\right| \geq k+1\right\}}\left|u_{n}\right|^{r-2} u_{n} T_{1}\left(u_{n}-T_{k}\left(u_{n}\right)\right) \mathrm{d} x \\
\geq & \int_{B_{k}}\left|u_{n}\right|^{r-2} u_{n} T_{1}\left(u_{n}-T_{k}\left(u_{n}\right)\right) \mathrm{d} x .
\end{aligned}
$$

Thus we have

$$
\begin{aligned}
\int_{B_{k}} \frac{\alpha\left|\nabla u_{n}\right|^{p}}{\left(1+\left|u_{n}\right|\right)^{\theta(p-1)} \mathrm{d} x+} & \leq\|f\|_{1}+1-\int_{B_{k}} b\left|u_{n}\right|^{r-2} u_{n} T_{1}\left(u_{n}-T_{k}\left(u_{n}\right)\right) \mathrm{d} x \\
& \leq\|f\|_{1}+1+\int_{B_{k}} b\left|u_{n}\right|^{r-1} \mathrm{~d} x
\end{aligned}
$$




$$
\leq C\left(1+\left(\int_{B_{k}}\left|u_{n}\right|^{q^{*}} \mathrm{~d} x\right)^{\frac{r-1}{q^{*}}}\left|B_{k}\right|^{1-\frac{r-1}{q^{*}}}\right),
$$

where $q$ is given in (8) and $q^{*}=\frac{N q}{N-q}$.

Let $s=\frac{q \theta(p-1)}{p}$. Note that $q<p$ and $\frac{p s}{p-q}<q^{*}$. For $\forall k>0$, we estimate $\int_{B_{k}}\left|\nabla u_{n}\right|^{q} \mathrm{~d} x$ as follows:

$$
\begin{aligned}
& \int_{B_{k}}\left|\nabla u_{n}\right|^{q} \mathrm{~d} x=\int_{B_{k}} \frac{\left|\nabla u_{n}\right|^{q}}{\left(1+\left|u_{n}\right|\right)^{s}} \cdot\left(1+\left|u_{n}\right|\right)^{s} \mathrm{~d} x \\
& \leq\left(\int_{B_{k}} \frac{\left|\nabla u_{n}\right|^{p}}{\left(1+\left|u_{n}\right|\right)^{\theta(p-1)}} \mathrm{d} x\right)^{\frac{q}{p}}\left(\int_{B_{k}}\left(1+\left|u_{n}\right|\right)^{\frac{p s}{p-q}} \mathrm{~d} x\right)^{\frac{p-q}{p}} \\
& \leq C\left(\int_{B_{k}} \frac{\left|\nabla u_{n}\right|^{p}}{\left(1+\left|u_{n}\right|\right)^{\theta(p-1)}} \mathrm{d} x\right)^{\frac{q}{p}}\left(\left|B_{k}\right|^{\frac{p-q}{p}}+\left(\int_{B_{k}}\left|u_{n}\right|^{\frac{p s}{p-q}} \mathrm{~d} x\right)^{\frac{p-q}{p}}\right) \\
& \leq C\left(\int_{B_{k}} \frac{\left|\nabla u_{n}\right|^{p}}{\left(1+\left|u_{n}\right|\right)^{\theta(p-1)}} \mathrm{d} x\right)^{\frac{q}{p}}\left(\left|B_{k}\right|^{\frac{p-q}{p}}+\left(\int_{B_{k}}\left|u_{n}\right|^{q^{*}} \mathrm{~d} x\right)^{\frac{s}{q^{*}}}\left|B_{k}\right|^{\frac{p-q}{p}-\frac{s}{q^{*}}}\right) \\
& \leq C\left(\left|B_{k}\right|^{\frac{p-q}{p}}+\left|B_{k}\right|^{\frac{p-q}{p}-\frac{s}{q^{*}}}\left(\int_{B_{k}}\left|u_{n}\right|^{q^{*}} \mathrm{~d} x\right)^{\frac{s}{q^{*}}}+\left|B_{k}\right|^{1-p_{1}}\left(\int_{B_{k}}\left|u_{n}\right|^{q^{*}} \mathrm{~d} x\right)^{p_{1}}\right. \\
& \left.+\left|B_{k}\right|^{1-p_{2}}\left(\int_{B_{k}}\left|u_{n}\right|^{q^{*}} \mathrm{~d} x\right)^{p_{2}}\right) \text { by (25) } \\
& =C\left(\left|B_{k}\right|^{\frac{p-q}{p}}+\left|B_{k}\right|^{\frac{p-q}{p}-\frac{s}{q^{*}}}\left(\int_{B_{k}}\left|u_{n}\right|^{q^{*}} \mathrm{~d} x\right)^{\frac{s}{q^{*}}}\right. \\
& +\left|B_{k}\right|^{1-p_{1}-C_{1}}\left|B_{k}\right|^{C_{1}}\left(\int_{B_{k}}\left|u_{n}\right|^{q^{*}} \mathrm{~d} x\right)^{p_{1}} \\
& \left.+\left|B_{k}\right|^{1-p_{2}-C_{2}}\left|B_{k}\right|^{C_{2}}\left(\int_{B_{k}}\left|u_{n}\right|^{q^{*}} \mathrm{~d} x\right)^{p_{2}}\right),
\end{aligned}
$$

where $p_{1}=\frac{q}{p} \frac{r-1}{q^{*}}, p_{2}=\frac{s}{q^{*}}+\frac{q}{p} \frac{r-1}{q^{*}}, C_{1}$ and $C_{2}$ are positive constants to be chosen later.

Note that $\theta<\frac{p-r}{p-1}$, it follows

$$
\frac{\theta(p-1)}{p}+\frac{r-1}{p}<\frac{p-1}{p}<1-\frac{1}{N}=1-\frac{1}{q}+\frac{1}{q^{*}} .
$$

Thus

$$
\begin{aligned}
\frac{\theta q(p-1)}{p}+\frac{q(r-1)}{p}+1<q+\frac{q}{q^{*}} & \Leftrightarrow s+\frac{q(r-1)}{p}+1<q+\frac{q}{q^{*}} \\
& \Leftrightarrow p_{2}+\frac{1-p_{2}}{q^{*}+1}<\frac{q}{q^{*}} .
\end{aligned}
$$

Note that $p_{1}<p_{2}<1$. Then, for $i=1,2$, we always have

$$
p_{i}+\frac{1-p_{i}}{q^{*}+1}<\frac{q}{q^{*}}<1 .
$$


From this, we may find positive $C_{i}(i=1,2)$ such that

$$
p_{i}+\frac{1-p_{i}}{q^{*}+1}<p_{i}+C_{i}<\frac{q}{q^{*}}<1, \quad i=1,2 .
$$

It follows

$$
\frac{1-p_{i}}{q^{*}+1}<C_{i} \quad \Leftrightarrow \quad 1-p_{i}-C_{i}<C_{i} q^{*}, \quad i=1,2,
$$

which implies

$$
C_{i} \alpha_{i} q^{*}=\frac{C_{i} q^{*}}{1-p_{i}-C_{i}}>1, \quad i=1,2,
$$

with $\alpha_{i}=\frac{1}{1-p_{i}-C_{i}}>1, i=1,2$. Let $\beta_{i}=\frac{1}{p_{i}+C_{i}}>1, i=1,2$. Then we have $\frac{1}{\alpha_{i}}+\frac{1}{\beta_{i}}=1(i=1,2)$.

Since $\left|B_{k}\right| \leq \frac{1}{k^{q^{*}}} \int_{B_{k}}\left|u_{n}\right|^{q^{*}} \mathrm{~d} x,\left|B_{k}\right|^{1-p_{1}-C_{1}} \leq|\Omega|^{1-p_{1}-C_{1}}$, and $\left|B_{k}\right|^{1-p_{2}-C_{2}} \leq|\Omega|^{1-p_{2}-C_{2}}$, we have, for $k \geq k_{0} \geq 1$,

$$
\begin{aligned}
\int_{B_{k}}\left|\nabla u_{n}\right|^{q} \mathrm{~d} x \leq & \frac{C}{k^{q^{*}\left(\frac{p-q}{p}-\frac{s}{q^{*}}\right)}}\left(\int_{B_{k}}\left|u_{n}\right|^{q^{*}} \mathrm{~d} x\right)^{\frac{p-q}{p}} \\
& +\frac{C}{k^{q^{*} C_{1}}}\left(\int_{B_{k}}\left|u_{n}\right|^{q^{*}} \mathrm{~d} x\right)^{p_{1}+C_{1}}+\frac{C}{k^{q^{*} C_{2}}}\left(\int_{B_{k}}\left|u_{n}\right|^{q^{*}} \mathrm{~d} x\right)^{p_{2}+C_{2}} .
\end{aligned}
$$

Summing up from $k=k_{0}$ to $k=K$ and using Hölder's inequality, one has

$$
\begin{aligned}
& \sum_{k=k_{0}}^{K} \int_{B_{k}}\left|\nabla u_{n}\right|^{q} \mathrm{~d} x \leq C\left(\sum_{k=k_{0}}^{K} \frac{1}{k^{q^{*}\left(\frac{p-q}{p}-\frac{s}{q^{*}}\right) \frac{p}{q}}}\right)^{\frac{q}{p}} \cdot\left(\sum_{k=k_{0}}^{K} \int_{B_{k}}\left|u_{n}\right|^{q^{*}} \mathrm{~d} x\right)^{\frac{p-q}{p}} \\
& +C\left(\sum_{k=k_{0}}^{K} \frac{1}{k^{q^{*} C_{1} \alpha_{1}}}\right)^{\frac{1}{\alpha_{1}}} \cdot\left(\sum_{k=k_{0}}^{K}\left(\int_{B_{k}}\left|u_{n}\right|^{q^{*}} \mathrm{~d} x\right)^{\beta_{1}\left(p_{1}+C_{1}\right)}\right)^{\frac{1}{\beta_{1}}} \\
& +C\left(\sum_{k=k_{0}}^{K} \frac{1}{k^{q^{*} C_{2} \alpha_{2}}}\right)^{\frac{1}{\alpha_{2}}} \cdot\left(\sum_{k=k_{0}}^{K}\left(\int_{B_{k}}\left|u_{n}\right|^{q^{*}} \mathrm{~d} x\right)^{\beta_{2}\left(p_{2}+C_{2}\right)}\right)^{\frac{1}{\beta_{2}}} \\
& =C\left(\sum_{k=k_{0}}^{K} \frac{1}{k^{q^{*}\left(\frac{p-q}{p}-\frac{s}{q^{*}}\right) \frac{p}{q}}}\right)^{\frac{q}{p}} \cdot\left(\sum_{k=k_{0}}^{K} \int_{B_{k}}\left|u_{n}\right|^{q^{*}} \mathrm{~d} x\right)^{\frac{p-q}{p}} \\
& +C\left(\sum_{k=k_{0}}^{K} \frac{1}{k^{q^{*} C_{1} \alpha_{1}}}\right)^{\frac{1}{\alpha_{1}}} \cdot\left(\sum_{k=k_{0}}^{K} \int_{B_{k}}\left|u_{n}\right|^{q^{*}} \mathrm{~d} x\right)^{p_{1}+C_{1}} \\
& +C\left(\sum_{k=k_{0}}^{K} \frac{1}{k^{q^{*} C_{2} \alpha_{2}}}\right)^{\frac{1}{\alpha_{2}}} \cdot\left(\sum_{k=k_{0}}^{K} \int_{B_{k}}\left|u_{n}\right|^{q^{*}} \mathrm{~d} x\right)^{p_{2}+C_{2}} .
\end{aligned}
$$

Note that

$$
\int_{\left\{\left|u_{n}\right| \leq K\right\}}\left|\nabla u_{n}\right|^{q} \mathrm{~d} x=\int_{D_{k_{0}}}\left|\nabla u_{n}\right|^{q} \mathrm{~d} x+\sum_{k=k_{0}}^{K} \int_{B_{k}}\left|\nabla u_{n}\right|^{q} \mathrm{~d} x .
$$


To estimate the first integral in the right-hand side of (29), we compute by using Hölder's inequality and (24), obtaining

$$
\begin{aligned}
\int_{D_{k_{0}}}\left|\nabla u_{n}\right|^{q} \mathrm{~d} x & \leq\left(\int_{D_{k_{0}}} \frac{\left|\nabla u_{n}\right|^{p}}{\left(1+\left|u_{n}\right|\right)^{\theta(p-1)}} \mathrm{d} x\right)^{\frac{q}{p}}\left(\int_{D_{k_{0}}}\left(1+\left|u_{n}\right|\right)^{\frac{p s}{p-q}} \mathrm{~d} x\right)^{\frac{p-q}{p}} \\
& \leq C
\end{aligned}
$$

where $C$ depends only on $\alpha, \beta, b, p, \theta,\|j\|_{p^{\prime}},\|f\|_{1},\left\|\nabla v_{0}\right\|_{p},\left\|v_{0}\right\|_{\infty}$, and $k_{0}$.

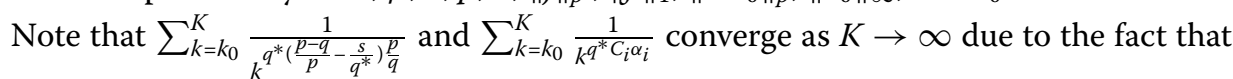
$q^{*}\left(\frac{p-q}{p}-\frac{s}{q^{*}}\right) \frac{p}{q}>1$ and $q^{*} C_{i} \alpha_{i}>1$ by (27), respectively. Combining (28)-(30), we get for $k_{0}$ large enough

$$
\begin{aligned}
\int_{\left\{\left|u_{n}\right| \leq K\right\}}\left|\nabla u_{n}\right|^{q} \mathrm{~d} x \leq & C+C\left(\int_{\left\{\left|u_{n}\right| \leq K\right\}}\left|u_{n}\right|^{q^{*}} \mathrm{~d} x\right)^{\frac{p-q}{p}} \\
& +C\left(\int_{\left\{\left|u_{n}\right| \leq K\right\}}\left|u_{n}\right|^{q^{*}} \mathrm{~d} x\right)^{p_{1}+C_{1}} \\
& +C\left(\int_{\left\{\left|u_{n}\right| \leq K\right\}}\left|u_{n}\right|^{q^{*}} \mathrm{~d} x\right)^{p_{2}+C_{2}} .
\end{aligned}
$$

Since $p>q, T_{K}\left(u_{n}\right) \in W^{1, q}(\Omega), T_{K}(g)=g \in W^{1, q}(\Omega)$ for $K>\|g\|_{\infty}$. Hence $T_{K}\left(u_{n}\right)-g \in$ $W_{0}^{1, q}(\Omega)$. Using the Sobolev embedding $W_{0}^{1, q}(\Omega) \subset L^{q^{*}}(\Omega)$ and Poincaré's inequality, we obtain

$$
\begin{aligned}
\left\|T_{K}\left(u_{n}\right)\right\|_{q^{*}}^{q} & \leq 2^{q-1}\left(\left\|T_{K}\left(u_{n}\right)-g\right\|_{q^{*}}^{q}+\|g\|_{q^{*}}^{q}\right) \\
& \leq C\left(\left\|\nabla\left(T_{K}\left(u_{n}\right)-g\right)\right\|_{q}^{q}+\|g\|_{q^{*}}^{q}\right) \\
& \leq C\left(\left\|\nabla T_{K}\left(u_{n}\right)\right\|_{q}^{q}+\|\nabla g\|_{q}^{q}+\|g\|_{q^{*}}^{q}\right) \\
& \leq C\left(1+\int_{\left\{\left|u_{n}\right| \leq K\right\}}\left|\nabla u_{n}\right|^{q} \mathrm{~d} x\right) .
\end{aligned}
$$

Using the fact that

$$
\int_{\left\{\left|u_{n}\right| \leq K\right\}}\left|u_{n}\right|^{q^{*}} \mathrm{~d} x \leq \int_{\left\{\left|u_{n}\right| \leq K\right\}}\left|T_{K}\left(u_{n}\right)\right|^{q^{*}} \mathrm{~d} x \leq\left\|T_{K}\left(u_{n}\right)\right\|_{q^{*}}^{q^{*}}
$$

we obtain from (31)-(32)

$$
\begin{aligned}
\int_{\left\{\left|u_{n}\right| \leq K\right\}}\left|\nabla u_{n}\right|^{q} \mathrm{~d} x \leq & C+C\left(1+\int_{\left\{\left|u_{n}\right| \leq K\right\}}\left|\nabla u_{n}\right|^{q} \mathrm{~d} x\right)^{\frac{q^{*}}{q} \frac{p-q}{p}} \\
& +C\left(1+\int_{\left\{\left|u_{n}\right| \leq K\right\}}\left|\nabla u_{n}\right|^{q} \mathrm{~d} x\right)^{\left(p_{1}+C_{1}\right) \frac{q^{*}}{q}} \\
& +C\left(1+\int_{\left\{\left|u_{n}\right| \leq K\right\}}\left|\nabla u_{n}\right|^{q} \mathrm{~d} x\right)^{\left(p_{2}+C_{2}\right) \frac{q^{*}}{q}} .
\end{aligned}
$$


Note that $p<N \Leftrightarrow \frac{q^{*}}{q} \frac{p-q}{p}<1$ and $\left(p_{i}+C_{i}\right) \frac{q^{*}}{q}<1$ by (26). It follows from (34) that, for $k_{0}$ large enough, $\int_{\left\{\left|u_{n}\right| \leq K\right\}}\left|\nabla u_{n}\right|^{q} \mathrm{~d} x$ is bounded independently of $n$ and $K$. Using (32) and (33), we deduce that $\int_{\left\{\left|u_{n}\right| \leq K\right\}}\left|u_{n}\right|^{q^{*}} \mathrm{~d} x$ is also bounded independently of $n$ and $K$. Letting $K \rightarrow \infty$, we deduce that $\left\|\nabla u_{n}\right\|_{q}$ and $\left\|u_{n}\right\|_{q^{*}}$ are uniformly bounded independently of $n$. Particularly, $u_{n}$ is bounded in $W^{1, q}(\Omega)$. Therefore, there exist a subsequence of $\left\{u_{n}\right\}$ and a function $v \in W^{1, q}(\Omega)$ such that $u_{n} \rightarrow v$ weakly in $W^{1, q}(\Omega), u_{n} \rightarrow v$ strongly in $L^{q}(\Omega)$ and a.e. in $\Omega$. By Lemma 3, $u_{n} \rightarrow u$ in measure in $\Omega$, we conclude that $u=v$ and $u \in W^{1, q}(\Omega)$.

Lemma 6 There exist a subsequence of $\left\{u_{n}\right\}$ and a measurable function $u$ such that $\nabla u_{n}$ converges almost everywhere in $\Omega$ to $\nabla u$.

Proof Define $A(x, u, \xi)=\frac{a(x, \xi)}{\left(1+|u|^{(p-1)}\right.}$ (for the sake of simplicity, we omit the dependence of $A(x, u, \xi)$ on $x)$. Let $h>0, k>\max \left\{\|g\|_{\infty},\|\psi\|_{\infty}\right\}$, and $n \geq h+k$. Take $T_{k}(u)$ as a test function for (7), obtaining

$$
I_{7}(n, k, h) \leq \int_{\Omega} f_{n} T_{h}\left(u_{n}-T_{k}(u)\right) \mathrm{d} x+\int_{\Omega} b\left|u_{n}\right|^{r-2} u_{n} T_{h}\left(u_{n}-T_{k}(u)\right) \mathrm{d} x,
$$

where

$$
I_{7}(n, k, h)=\int_{\Omega} A\left(u_{n}, \nabla u_{n}\right) \cdot \nabla T_{h}\left(u_{n}-T_{k}(u)\right) \mathrm{d} x .
$$

Note that $r-1<q^{*}$, and $\int_{\Omega}\left|u_{n}\right|^{q^{*}} \mathrm{~d} x$ is uniformly bounded (see the proof of Lemma 5), thus $\left|u_{n}\right|$ converges strongly in $L^{1}(\Omega)$. Therefore we have

$$
\lim _{n \rightarrow \infty} \int_{\Omega}\left|u_{n}\right|^{r-2} u_{n} T_{h}\left(u_{n}-T_{k}(u)\right) \mathrm{d} x=\int_{\Omega}|u|^{r-2} u T_{h}\left(u-T_{k}(u)\right) \mathrm{d} x .
$$

Then, using the strong convergence of $f_{n}$ in $L^{1}(\Omega)$, one has

$$
\lim _{n \rightarrow \infty} I_{7}(n, k, h) \leq \int_{\Omega}-f T_{h}\left(u-T_{k}(u)\right) \mathrm{d} x+\int_{\Omega} b|u|^{r-2} u T_{h}\left(u-T_{k}(u)\right) \mathrm{d} x .
$$

It follows

$$
\lim _{k \rightarrow \infty} \lim _{n \rightarrow \infty} I_{7}(n, k, h) \leq 0 .
$$

Thanks to Lemma 3 and Lemma 5, we can proceed exactly as [19, Lemma 6] to conclude that, up to subsequence, $\nabla u_{n} \rightarrow \nabla u$ a.e.

Proof of Proposition 4. We shall prove that $\nabla u_{n}$ converges strongly to $\nabla u$ in $L^{q}(\Omega)$ for each $q$ being given by (8). To do that,we will apply Vitali's theorem, using the fact that by Lemma $5, \nabla u_{n}$ is bounded in $L^{q}(\Omega)$ for each $q$ given by (8). So let $s \in\left(q, \frac{N(p-1)(1-\theta)}{N-1-\theta(p-1)}\right)$ and $E \subset \Omega$ be a measurable set. Then we have by Hölder's inequality

$$
\int_{E}\left|\nabla u_{n}\right|^{q} \mathrm{~d} x \leq\left(\int_{E}\left|\nabla u_{n}\right|^{r} \mathrm{~d} x\right)^{\frac{q}{s}} \cdot|E|^{\frac{s-q}{s}} \leq C|E|^{\frac{s-q}{s}} \rightarrow 0
$$


uniformly in $n$, as $|E| \rightarrow 0$. From this and from Lemma 6, we deduce that $\nabla u_{n}$ converges strongly to $\nabla u$ in $L^{q}(\Omega)$.

Now assume that $0 \leq \theta<\min \left\{\frac{1}{N-p+1}, \frac{N}{N-1}-\frac{1}{p-1}, \frac{p-r}{p-1}\right\}$. Note that since $\nabla u_{n}$ converges to $\nabla u$ a.e. in $\Omega$, to prove the convergence

$$
\frac{a\left(x, \nabla u_{n}\right)}{\left(1+\left|u_{n}\right|\right)^{\theta(p-1)}} \rightarrow \frac{a(x, \nabla u)}{(1+|u|)^{\theta(p-1)}} \quad \text { strongly in }\left(L^{1}(\Omega)\right)^{N},
$$

it suffices, thanks to Vitali's theorem, to show that, for every measurable subset $E \subset \Omega$, $\int_{E}\left|\frac{a\left(x, \nabla u_{n}\right)}{\left(1+\left|u_{n}\right|\right)^{\theta(p-1)}}\right| \mathrm{d} x$ converges to 0 uniformly in $n$, as $|E| \rightarrow 0$. Note that $\left.p-1<\frac{N(p-1)(1-\theta)}{N-1-\theta(p-1)}\right)$ by assumptions. For any $q \in\left(p-1, \frac{N(p-1)(1-\theta)}{N-1-\theta(p-1)}\right)$, we deduce by Hölder's inequality

$$
\begin{aligned}
& \int_{E}\left|\frac{a\left(x, \nabla u_{n}\right)}{\left(1+\left|u_{n}\right|\right)^{\theta(p-1)}}\right| \mathrm{d} x \leq \beta \int_{E}\left(j+\left|\nabla u_{n}\right|^{p-1}\right) \mathrm{d} x \\
& \leq \beta\|j\|_{p^{\prime}}|E|^{\frac{1}{p}}+\beta\left(\int_{E}\left|\nabla u_{n}\right|^{q} \mathrm{~d} x\right)^{\frac{p-1}{q}}|E|^{\frac{q-p+1}{q}} \\
& \rightarrow 0 \quad \text { uniformly in } n \text { as }|E| \rightarrow 0 \text {. }
\end{aligned}
$$

Lemma 7 There exists a subsequence of $\left\{u_{n}\right\}$ such that, for all $k>0$,

$$
\frac{a\left(x, \nabla T_{k}\left(u_{n}\right)\right)}{\left(1+\left|T_{k}\left(u_{n}\right)\right|\right)^{\theta(p-1)}} \rightarrow \frac{a\left(x, \nabla T_{k}(u)\right)}{\left(1+\left|T_{k}(u)\right|\right)^{\theta(p-1)}} \quad \text { strongly in }\left(L^{1}(\Omega)\right)^{N} .
$$

Proof See the proof of [19, Lemma 7].

\section{Proof of the main result}

Now we have gathered all the lemmas needed to prove the existence of an entropy solution to the obstacle problem associated with $(f, \psi, g)$. In this part, let $f_{n}$ be a sequence of smooth functions converging strongly to $f$ in $L^{1}(\Omega)$, with $\left\|f_{n}\right\|_{1} \leq\|f\|_{1}+1$. We consider the sequence of approximated obstacle problems associated with $\left(f_{n}, \psi, g\right)$. The proof can be proceeded in the same way as in [8] and [19]. We provide details for readers' convenience.

Proof of Theorem 1 Let $v \in K_{g, \psi} \cap L^{\infty}(\Omega)$. Taking $v$ as a test function in (7) associated with $\left(f_{n}, \psi, g\right)$, we get

$$
\begin{aligned}
& \int_{\Omega} \frac{a\left(x, \nabla u_{n}\right)}{\left(1+\left|u_{n}\right|\right)^{\theta(p-1)}} \cdot \nabla\left(T_{t}\left(u_{n}-v\right)\right) \mathrm{d} x+\int_{\Omega} b\left|u_{n}\right|^{r-2} u_{n} T_{t}\left(u_{n}-v\right) \mathrm{d} x \\
& \quad \leq \int_{\Omega} f_{n} T_{t}\left(u_{n}-v\right) \mathrm{d} x .
\end{aligned}
$$

Since $\left\{\left|u_{n}-v\right|<t\right\} \subset\left\{\left|u_{n}\right|<s\right\}$ with $s=t+\|v\|_{\infty}$, the previous inequality can be written as

$$
\begin{aligned}
\int_{\Omega} \chi_{n} \nabla_{A} T_{s}\left(u_{n}\right) \cdot \nabla v \mathrm{~d} x \geq & \int_{\Omega}-f_{n} T_{t}\left(u_{n}-v\right) \mathrm{d} x+\int_{\Omega} b\left|u_{n}\right|^{r-2} u_{n} T_{t}\left(u_{n}-v\right) \mathrm{d} x \\
& +\int_{\Omega} \chi_{n} \nabla_{A} T_{s}\left(u_{n}\right) \cdot \nabla T_{s}\left(u_{n}\right) \mathrm{d} x,
\end{aligned}
$$


where $\chi_{n}=\chi_{\left\{\left|u_{n}-v\right|<t\right\}}$ and $\nabla_{A} u=\frac{a(x, \nabla u)}{(1+|u|)^{\theta(p-1)}}$. It is clear that $\chi_{n} \rightarrow \chi$ weakly* in $L^{\infty}(\Omega)$. Moreover, $\chi_{n}$ converges a.e. to $\chi_{\{|u-v|<t\}}$ in $\Omega \backslash\{|u-v|=t\}$. It follows that

$$
\chi= \begin{cases}1, & \text { in }\{|u-v|<t\} \\ 0, & \text { in }\{|u-v|>t\}\end{cases}
$$

Note that we have $\mathcal{L}^{N}(\{|u-v|=t\})=0$ for a.e. $t \in(0, \infty)$. So there exists a measurable set $\mathcal{O} \subset(0, \infty)$ such that $\mathcal{L}^{N}(\{|u-v|=t\})=0$ for all $t \in(0, \infty) \backslash \mathcal{O}$. Assume that $t \in(0, \infty) \backslash$ $\mathcal{O}$. Then $\chi_{n}$ converges weakly* in $L^{\infty}(\Omega)$ and a.e. in $\Omega$ to $\chi=\chi_{\{|u-v|<t\}}$. Since $\nabla T_{s}\left(u_{n}\right)$ converges a.e. to $\nabla T_{s}(u)$ in $\Omega$ (Proposition 4 ), we obtain by Fatou's lemma

$$
\liminf _{n \rightarrow \infty} \int_{\Omega} \chi_{n} \nabla_{A} T_{s}\left(u_{n}\right) \cdot \nabla T_{s}\left(u_{n}\right) \mathrm{d} x \geq \int_{\Omega} \chi \nabla_{A} T_{s}(u) \cdot \nabla T_{s}(u) \mathrm{d} x .
$$

Using the strong convergence of $\nabla_{A} T_{s}\left(u_{n}\right)$ to $\nabla_{A} T_{s}(u)$ in $L^{1}(\Omega)$ (Lemma 7) and the weak" convergence of $\chi_{n}$ to $\chi$ in $L^{\infty}(\Omega)$, we obtain

$$
\lim _{n \rightarrow \infty} \int_{\Omega} \chi_{n} \nabla_{A} T_{s}\left(u_{n}\right) \cdot \nabla v \mathrm{~d} x=\int_{\Omega} \chi \nabla_{A} T_{s}(u) \cdot \nabla v \mathrm{~d} x
$$

Moreover, due to the strong convergence of $f_{n}$ to $f$ and $\left|u_{n}\right|^{r-2} u_{n}$ to $|u|^{r-2} u$ (by $r-1<q^{*}$ and the boundedness of $\left.\left\|u_{n}\right\|_{q^{*}}\right)$ in $L^{1}(\Omega)$, and the weak* convergence of $T_{t}\left(u_{n}-v\right)$ to $T_{t}(u-v)$ in $L^{\infty}(\Omega)$, by passing to the limit in (35) and taking into account (36)-(37), we obtain

$$
\begin{aligned}
\int_{\Omega} \chi \nabla_{A} T_{s}(u) \cdot \nabla v \mathrm{~d} x-\int_{\Omega} \chi \nabla_{A} T_{s}(u) \cdot \nabla T_{s}(u) \mathrm{d} x \geq & \int_{\Omega}-f T_{t}(u-v) \mathrm{d} x \\
& +\int_{\Omega} b|u|^{r-2} u T_{t}(u-v) \mathrm{d} x
\end{aligned}
$$

which can be written as

$$
\begin{aligned}
\int_{\{|v-u| \leq t\}} \chi \nabla_{A} T_{s}(u) \cdot(\nabla v-\nabla u) \mathrm{d} x \geq & \int_{\Omega}-f T_{t}(u-v) \mathrm{d} x \\
& +\int_{\Omega} b|u|^{r-2} u T_{t}(u-v) \mathrm{d} x,
\end{aligned}
$$

or since $\chi=\chi_{\{|u-v|<t\}}$ and $\nabla\left(T_{t}(u-v)\right)=\chi_{\{|u-v|<t\}} \nabla(u-v)$

$$
\begin{gathered}
\int_{\Omega} \nabla_{A} u \cdot \nabla T_{t}(u-v) \mathrm{d} x+\int_{\Omega} b|u|^{r-2} u T_{t}(u-v) \mathrm{d} x \\
\quad \leq \int_{\Omega} f T_{t}(u-v) \mathrm{d} x, \forall t \in(0, \infty) \backslash \mathcal{O} .
\end{gathered}
$$

For $t \in \mathcal{O}$, we know that there exists a sequence $\left\{t_{k}\right\}$ of numbers in $(0, \infty) \backslash \mathcal{O}$ such that $t_{k} \rightarrow t$ due to $|\mathcal{O}|=0$. Therefore, we have

$$
\int_{\Omega} \nabla_{A} u \cdot \nabla T_{t_{k}}(u-v) \mathrm{d} x+\int_{\Omega} b|u|^{r-2} u T_{t_{k}}(u-v) \mathrm{d} x \leq \int_{\Omega} f T_{t_{k}}(u-v) \mathrm{d} x .
$$


Since $\nabla(u-v)=0$ a.e. in $\{|u-v|=t\}$, the left-hand side of (38) can be written as

$$
\int_{\Omega} \nabla_{A} u \cdot \nabla T_{t_{k}}(u-v) \mathrm{d} x=\int_{\Omega \backslash\{|u-v|=t\}} \chi_{\left\{|u-v|<t_{k}\right\}} \nabla_{A} u \cdot \nabla(u-v) \mathrm{d} x .
$$

The sequence $\chi_{\left\{|u-v|<t_{k}\right\}}$ converges to $\chi_{\{|u-v|<t\}}$ a.e. in $\Omega \backslash\{|u-v|=t\}$ and therefore converges weakly* in $L^{\infty}(\Omega \backslash\{|u-v|=t\})$. We obtain

$$
\begin{aligned}
\lim _{k \rightarrow \infty} \int_{\Omega} \nabla_{A} u \cdot \nabla T_{t_{k}}(u-v) \mathrm{d} x & =\int_{\Omega \backslash\{|u-v|=t\}} \chi_{\{|u-v|<t\}} \nabla_{A} u \cdot \nabla(u-v) \mathrm{d} x \\
& =\int_{\Omega} \chi_{\{|u-v|<t\}} \nabla_{A} u \cdot \nabla(u-v) \mathrm{d} x \\
& =\int_{\Omega} \nabla_{A} u \cdot \nabla T_{t}(u-v) \mathrm{d} x .
\end{aligned}
$$

For the right-hand side of (38), we have

$$
\left|\int_{\Omega} f T_{t_{k}}(u-v) \mathrm{d} x-\int_{\Omega} f T_{t}(u-v) \mathrm{d} x\right| \leq\left|t_{k}-t\right| \cdot\|f\|_{1} \rightarrow 0 \quad \text { as } k \rightarrow \infty .
$$

Similarly, we have

$$
\begin{aligned}
\left.\left|\int_{\Omega}\right| u\right|^{r-2} u T_{t_{k}}(u-v) \mathrm{d} x-\int_{\Omega}|u|^{r-2} u T_{t}(u-v) \mathrm{d} x \mid & \leq\left|t_{k}-t\right| \cdot\left\||u|^{r-1}\right\|_{1} \\
& \rightarrow 0 \quad \text { as } k \rightarrow \infty
\end{aligned}
$$

It follows from (38)-(41) that we have the inequality

$$
\begin{aligned}
& \int_{\Omega} \nabla_{A} u \cdot \nabla T_{t}(u-v) \mathrm{d} x+\int_{\Omega} b|u|^{r-2} u T_{t}(u-v) \mathrm{d} x \\
& \quad \leq \int_{\Omega} f T_{t}(u-v) \mathrm{d} x, \quad \forall t \in(0, \infty) .
\end{aligned}
$$

Hence, $u$ is an entropy solution of the obstacle problem associated with $(f, \psi, g)$. The dependence of the entropy solution on the data $f \in L^{1}(\Omega)$ is guaranteed by Proposition 4 .

Acknowledgements

The author would like to thank the reviewers for their valuable suggestions and comments to improve the quality of this paper.

\section{Funding}

This research was partially supported by the Fundamental Research Funds for the Central Universities: No. 2682019LK01.

Availability of data and materials

Not applicable.

Competing interests

The author declares that he has no competing interests.

Authors' contributions

This paper was completed by JZ independently. All authors read and approved the final manuscript.

\section{Publisher's Note}

Springer Nature remains neutral with regard to jurisdictional claims in published maps and institutional affiliations.

Received: 27 August 2018 Accepted: 14 July 2019 Published online: 24 July 2019 


\section{References}

1. Alvino, A., Boccardo, L., Ferone, V., Orsina, L., Trombetti, G.: Existence results for nonlinear elliptic equations with degenerate coercivity. Ann. Mat. Pura Appl. 182(1), 53-79 (2003)

2. Alvino, A., Ferone, V., Trombetti, G.: A priori estimates for a class of non uniformly elliptic equations. Atti Semin. Mat. Fis. Univ. Modena 46(suppl.), 381-391 (1998)

3. Bénilan, P., Boccardo, L., Gallouët, T., Gariepy, R., Pierre, M., Vazquez, J.L.: An L'1 theory of existence and uniqueness of nonlinear elliptic equations. Ann. Sc. Norm. Super. Pisa, Cl. Sci. 22(2), 240-273 (1995)

4. Boccardo, L., Brezis, H.: Some remarks on a class of elliptic equations with degenerate coercivity. Boll. Unione Mat. Ital., B 6(3), 521-530 (2003)

5. Boccardo, L., Cirmi, G.R.: Existence and uniqueness of solution of unilateral problems with $L^{1}$ data. J. Convex Anal. 6(1), 195-206 (1999)

6. Boccardo, L., Croce, G., Orsina, L.: Existence of solutions for some noncoercive elliptic problems involving deriratives of nonlinear terms. Differ. Equ. Appl. 4(1), 3-9 (2012)

7. Boccardo, L., Dall'Aglio, A., Orsina, L.: Existence and regularity results for some elliptic equations with degenerate coercivity. Atti Semin. Mat. Fis. Univ. Modena 46(suppl.), 51-81 (1998)

8. Challal, S., Lyaghfouri, A., Rodrigues, J.F.: On the A-obstacle problem and the Hausdorff measure of its free boundary. Ann. Mat. Pura Appl. 191(1), 113-165 (2012)

9. Chen, G.: Nonlinear elliptic equation with lower order term and degenerate coercivity. Math. Notes 93(1-2), 224-237 (2013)

10. Croce, G.: The regularizing effects of some lower order terms in an elliptic equation with degenerate coercivity. Rend. Mat. Appl. 27(7), 299-314 (2007)

11. Della Pietra, F., di Blasio, G.: Comparison, existence and regularity results for a class of nonuniformly elliptic equations. Differ. Equ. Appl. 2(1), 79-103 (2010)

12. Giachetti, D., Porzio, M.M.: Existence results for some non uniformly elliptic equations with irregular data. J. Math. Anal. Appl. 257(1), 100-130 (2001)

13. Giachetti, D., Porzio, M.M.: Elliptic equations with degenerate coercivity, gradient regularity. Acta Math. Sin. 19(2), 349-370 (2003)

14. Leray, J., Lions, J.L.: Quelques résultats de Višik sur les problèmes elliptiques nonlinéaires par les méthodes de Minty-Browder. Bull. Soc. Math. Fr. 93, 97-107 (1965)

15. Li, Z., Gao, W.: Existence results to a nonlinear $p(x)$-Laplace equation with degenerate coercivity and zero-order term: renormalized and entropy solutions. Appl. Anal. 95(2), 373-389 (2016)

16. Porretta, A.: Uniqueness and homogenization for a class of noncoercive operators in divergence form. Atti Semin. Mat. Fis. Univ. Modena 46, 915-936 (1998)

17. Stampacchia, G.: Le problème de Dirichlet pour les équations elliptiques du second ordre coefficients discontinus. Ann. Inst. Fourier (Grenoble) 15(1), 189-258 (1965)

18. Zeidler, E.: Nonlinear Functional Analysis and Its Applications. II, B: Nonlinear Monotone Operators. Springer, New York (1990) (translated from the German by the author and F. Leo)

19. Zheng, J., Tavares, L.S.: The obstacle problem for nonlinear noncoercive elliptic equations with $L^{1}$-data. Bound. Value Probl. 2019, 53 (2019). https://doi.org/10.1186/s13661-019-1168-2

\section{Submit your manuscript to a SpringerOpen ${ }^{\circ}$ journal and benefit from:}

- Convenient online submission

- Rigorous peer review

- Open access: articles freely available online

- High visibility within the field

- Retaining the copyright to your article

Submit your next manuscript at $>$ springeropen.com 